\title{
Mulheres profissionais da saúde e as repercussões da pandemia da Covid-19: é mais difícil para elas?
}

\author{
Female health care professionals and repercussions of the Covid-19 \\ pandemics: is it harder for them?
}

Julia Vieira', Isabela Anido', Karina Calife'

DOI: $10.1590 / 0103-1104202213203$

RESUMO A pandemia da Covid-19 promoveu mudanças significativas no cotidiano de todos, mas entre os profissionais da saúde, estas foram ainda mais profundas. A crise sanitária impactou relações pessoais, sociais e familiares, trazendo sensação de desamparo e insegurança, agravadas ainda pelo desastroso enfrentamento da pandemia pelo governo federal no Brasil. A força de trabalho na saúde tem sido apontada pela literatura como majoritariamente feminina e o papel social de gênero, somado ao contexto atual, agrava as implicações da pandemia para as mulheres. Este estudo analisou as repercussões da pandemia da Covid-19 sobre diferentes perfis de profissionais da saúde no estado de São Paulo. Estudo descritivo, utilizou questionário semiestruturado, on-line validado e o processamento dos dados quantitativos foi realizado pelo software Stata 13.0. Foram comparadas as respostas de acordo com o gênero declarado. Os achados corroboram com a literatura acerca da prevalência importante de sobrecarga de profissionais da saúde, e da discrepância entre fatores de sobrecarga apontados por gênero. A sobrecarga doméstica foi mais presente entre mulheres, reforçando que estas são afetadas de maneira desigual. Essa situação fragiliza a saúde mental, traz alterações no humor, sono, cognição, ansiedade, desconforto físico e pessimismo. Os resultados reforçam a necessidade de um olhar de gênero para as ações e respostas às consequências que surgirão à medida que a pandemia avança e na recuperação da sociedade no pós-pandemia.

PALAVRAS-CHAVES Covid-19. Pandemias. Pessoal da saúde. Gênero e saúde. Saúde mental.

1 Faculdade de Ciências Médicas da Santa Casa de São Paulo (FCMSCSP) São Paulo (SP), Brasil. juliargvieira@hotmail.com

\begin{abstract}
The Covid-19 pandemic promoted significant changes in everyone's daily lives, but among health professionals, those were even more profound. It impacted personal, social and family relationships, bringing feelings of helplessness and insecurity, aggravated by the disastrous confrontation of the pandemic by the Federal Government in Brazil. The health workforce has been identified in the literature as mostly female. The social role of gender, added to the current context, aggravates the implications of the pandemic for women. This study analyzed the repercussions of the Covid-19 pandemic on different profiles of health professionals in the State of São Paulo. This is a descriptive study which used a semi-structured, online, validated questionnaire. The processing of quantitative data was made using Stata 13.0 software. Answers were compared according to the declared gender. The findings corroborate the literature on the prevalence of significant burden on health professionals and the discrepancy between burden factors identified by gender. Household overload was more present among women, reinforcing that they are unevenly affected. This situation weakens mental health, brings changes in mood, sleep, cognition, anxiety, physical discomfort, and pessimism. Our results reinforce the need for a gender perspective in actions and responses to the consequences that will arise as the pandemic advances and in the recovery of society in the post-pandemic period.
\end{abstract}

KEYWORDS Covid-19. Pandemics. Health personnel. Gender and health. Mental health. 


\section{Introdução}

Declarada no dia 11 de março de 2020, pela Organização Mundial da Saúde (OMS) ${ }^{1}$, a pandemia causada pelo Sars-CoV-2, agente etiológico responsável pela doença Covid-19, afetou a vida das pessoas de diversas maneiras. Inicialmente, incertezas trazidas pelo vírus quanto à forma de transmissão, velocidade de disseminação e letalidade trouxeram enormes desafios no combate da Covid-19. Em um primeiro momento, mundo afora, foram estabelecidas medidas não farmacológicas na tentativa de conter a circulação do vírus, diminuindo sua transmissão. Medidas de prevenção à infecção, como o uso de máscaras, o distanciamento social, a higienização de mãos, a ventilação de ambientes, a ampla testagem para Covid19, lockdowns e fechamento de fronteiras foram adotadas. Essas medidas protetivas são estimuladas pela OMS até hoje ${ }^{2}$. Frente à crise sanitária instaurada no Brasil e a ainda baixa taxa de vacinação, até julho de 2021, as medidas não farmacológicas remanescem com grande importância na contenção do vírus.

Enquanto países da Europa e outros, como a Nova Zelândia, controlaram a pandemia precocemente ${ }^{3}$ com medidas de distanciamento, fechamento de fronteiras e uso obrigatório de máscaras, o Brasil teve um desempenho não apenas pífio, mas intencionalmente ineficiente no combate à pandemia. O país da Oceania reportou, desde o início da pandemia até julho de 2021, apenas 26 mortes $^{4}$. Já no Brasil, com as crenças negacionistas disseminadas pelo governo federal, a baixa adesão às medidas de isolamento e a desvalorização do uso de máscaras, tivemos uma das piores curvas epidemiológicas do mundo, com manutenção de altas médias móveis de casos e de óbitos por um longo período. Disputando os piores dados com países como Estados Unidos e Índia, o Brasil é hoje o terceiro pior país em número absoluto de casos registrados no mundo e o segundo no ranking de óbitos pela doença ${ }^{4}$.

A partir de então, a dificuldade de organização de estratégias de contenção da circulação do vírus e, atualmente, a condução nacional desestruturada e desarticulada da vacinação entre estados e municípios ${ }^{5}$, resultou na recente marca atingida de mais de 607.000 mortes pela Covid-194 no Brasil. Segundo o consórcio de imprensa, a partir da consolidação dos dados das secretarias estaduais de saúde, temos 21.793 .402 casos e 607.462 mortes até 30 de outubro de 2021.

A postura negacionista que permeou os dezenove meses do vírus no País, acrescida da falta de controle incisivo e da falta de uniformidade na condução da pandemia no Brasil, resultou em um grande desgaste mental da população $0^{6}$, dificultando ainda mais a contenção de comportamentos de risco para a infecção. $\mathrm{O}$ aumento das aglomerações e o baixo uso de máscaras são alguns exemplos de comportamentos que se tornaram comuns e dificultam o controle dos casos. Como na disseminação inicial do vírus no País, o descumprimento das medidas de distanciamento social perpetuou-se tanto nas elites, que voltaram a ocupar os leitos de hospitais particulares contaminados pela Covid-197, como nas classes sociais mais vulneráveis, com menor possibilidade de aderir a medidas como home office e isolamento social e que mantém o uso do transporte público ${ }^{8}$. No estado de São Paulo, estudos demonstraram que a região metropolitana apresentou a maior quantidade de casos positivos na pandemia da Covid-19. Além disso, as principais rodovias e aeroportos que conectam a capital às cidades com maior densidade populacional foram muito importantes para a disseminação dos casos, indicando uma expansão da capital para o interior - a 'interiorização' da pandemia9. Outro estudo observou que no município de São Paulo, por exemplo, existia importante diferença na soroprevalência do Sars-CoV-2 em distritos de renda média mais alta em relação aos de renda média mais baixa, chegando a $21,6 \%$ nos distritos mais ricos e $30,4 \%$ nos distritos mais pobres ${ }^{10}$ - a 'periferização' da pandemia.

Mesmo com a chegada das vacinas em janeiro de 2021, o cenário de crise sanitária 
pouco se alterou em decorrência da desorganização, em âmbito nacional, da estratégia de vacinação no País. Em julho de 2021, após a emergência de uma segunda onda de casos no País, sem que ainda tivéssemos controlado a primeira ${ }^{11,12}$, vimos a manutenção da transmissão do vírus. Em boa parte dos estados, apesar de leve melhora, a taxa de ocupação de leitos de Unidade de Terapia Intensiva (UTI) permaneceu em níveis críticos de março a junho deste ano, de acordo com o boletim extraordinário do Observatório Covid-19 da Fundação Oswaldo Cruz (Fiocruz), de 14 de julho de $2021^{12}$. Soma-se à intensificação da crise sanitária, a diminuição da adesão às medidas não farmacológicas para contenção e bloqueio da transmissão e a demora na vacinação, com somente $15,7 \%$ da população vacinada com ambas as doses da vacina e $43,2 \%$ com a primeira dose da vacina até julho de $2021^{13}$.

Algo importante é estarmos atentos à manutenção de praticamente a mesma força de trabalho desde 2020, início da pandemia, até hoje, com os profissionais já esgotados pelos desafios estabelecidos, com alta demanda de atendimento e com aumento do volume de trabalho em todas as esferas da saúde. Soma-se a esta sobrecarga a desinformação disseminada e a instabilidade política, a comunicação inadequada, a falta de Equipamentos de Proteção Individual (EPIs) para todos os profissionais de saúde, a falta de políticas públicas e de testagem adequada com acompanhamento dos casos positivos e suspeitos e do rastreamento dos comunicantes, o medo da infecção, o estigma profissional, o distanciamento de redes de suporte e o luto pela perda de pacientes ou até mesmo de familiares ${ }^{\mathbf{1 4}}$.

Sendo a linha de frente no combate à Covid19 majoritariamente feminina ${ }^{15} \mathrm{e}$ a área da saúde composta, predominantemente, por mulheres ${ }^{16}$, a pandemia as afeta de maneira desigual. Para profissionais do sexo feminino atuantes na área da saúde, a pandemia tem um peso maior em decorrência do papel de gênero da mulher na sociedade, levando à preocupação, exaustão física, alta carga mental e uma gama de consequências nas diferentes profissões. Simone de Beauvoir ${ }^{17}$ elucida em seu livro ' $\mathrm{O}$ Segundo Sexo' (1980) o debate acerca do papel da mulher na sociedade e como ele é construído com base num papel de gênero estabelecido.

Dentro do papel construído para a mulher, entra o debate acerca da divisão sexual do trabalho, abordado por Helena Hirata ${ }^{18}$. De acordo com a socióloga, essa divisão se baseia no princípio de que há a separação do trabalho, entre o homem e a mulher, em decorrência do sexo biológico, estruturando a distribuição diferencial dos dois gêneros no mercado de trabalho. Ademais, ela ampara a ideia de que há uma hierarquização nesta divisão, que denota o trabalho do homem como superior ao da mulher e resulta na repartição desigual do trabalho doméstico, que é previsto como de encargo feminino.

A socióloga Lícia Peres ${ }^{19}$ destaca que esta divisão assimétrica culmina no acúmulo de horas trabalhadas pela mulher como um todo: após a inserção da mulher no mercado de trabalho, ela assume o trabalho fora de casa, na esfera pública, bem como dentro de casa, na esfera privada (em geral, cuidados com a casa, crianças, enfermos), desdobrando-se em múltiplos papéis. A sobrecarga pela dupla jornada de trabalho feminina fica evidente quando olhamos os dados de 2020 do Instituto Brasileiro de Geografia e Estatística (IBGE) ${ }^{\mathbf{2 0}}$ que indicam que, em geral, as mulheres dedicam 10,4 horas a mais por semana que os homens nos afazeres domésticos, sendo essa taxa de realização de afazeres maior entre as mulheres independente de cor, raça ou idade.

Ainda, em sua teoria crítica sobre justiça social, a filósofa norte-americana Nancy Fraser analisa a redistribuição do trabalho entre o trabalho produtivo, definido como aquele que resulta em um salário, com valor de troca dentro do sistema capitalista e o trabalho reprodutivo, definido com aquele necessário para o sustento da vida humana na esfera doméstica, sem geração de riqueza, realizado pela mulher ${ }^{21}$. Fraser ${ }^{22}$ sustenta a teoria de que parte da injustiça econômica que envolve as mulheres deriva da sustentação econômica de 
que o trabalho reprodutivo (gratuito, feminino e desvalorizado socialmente) provê ao do trabalho produtivo realizado pelos homens. É bem estabelecido, portanto, o impacto do gênero nas dinâmicas de trabalho na sociedade atual.

Antes da pandemia, o resultado da inserção feminina no mercado de trabalho era a terceirização do trabalho doméstico, na maioria das vezes para outras mulheres de menor renda e em geral pretas e pardas ${ }^{23}$. O distanciamento social e a mudança desse cenário levaram à redução da presença das empregadas domésticas nas moradias. Na nova conjuntura, o excesso do trabalho doméstico aparenta recair para as mulheres na residência. Portanto, as mulheres da área da saúde, além de ganharem maior responsabilidade profissionalmente com o enfrentamento da pandemia, permanecem como cuidadoras principais do lar: elas encontram-se duplamente sobrecarregadas ${ }^{\mathbf{2 4}}$.

Atualmente, além dessa sobrecarga advinda da dupla jornada de trabalho, a presença diária do cenário nacional desgastante também contribui negativamente para suas angústias. No décimo sexto mês após a chegada do vírus ao País, o esgotamento e sobrecarga delas é uma realidade.

O maior volume de trabalho converte-se em desgaste e gera sobrecarga mental. Numa nova rotina em que se borram as fronteiras entre horário de trabalho e horário de lazer, a demanda pela disponibilidade constante das profissionais dentro e fora de casa gera sobrecarga que se soma ao estresse e ansiedade constante, devido ao contexto pandêmico e resulta em uma carga mental enorme com grande repercussão na saúde mental dessa população 25 .

Ainda, a realidade ditada pela presença da Covid-19 é regida pela alteração das relações pessoais, profissionais, familiares e sociais, devido ao distanciamento que o vírus determina $^{26}$ e seu impacto nas redes de suporte disponíveis para essas profissionais, que convivem com nova fonte de estresse e ansiedade rotineiramente ${ }^{25}$, lidando diariamente com questões da saúde no enfrentamento direto ou indireto à Covid-19.
Ainda não há como saber o impacto da pandemia na vida delas, visto que este é um cenário sem precedentes. Traçar o perfil das profissionais para entender o que as afeta nesse contexto é importante na compreensão das repercussões desse período na saúde dessas trabalhadoras, que ainda terão um longo caminho no combate à pandemia do coronavírus no País. Será que o impacto psicopatológico nessas pessoas estará entre as sequelas da Covid-19? Será que estará acentuado em meio aos indivíduos implicados no universo da saúde? Quanto será que o trabalho produtivo e reprodutivo impacta nisso? Será que é maior para elas? A literatura já aponta esse tipo de adoecimento decorrente de epidemias anteriores ${ }^{\mathbf{2 7}}$.

Esse artigo se propõe a analisar as repercussões da pandemia da Covid-19 entre diferentes perfis de profissionais da saúde no estado de São Paulo. Ainda, entender as repercussões entre as mulheres trabalhadoras da área da saúde, descrevendo e analisando a sobrecarga de trabalho e suas principais manifestações entre as entrevistadas. Pretende-se a, também, comparar com as principais repercussões apontadas pelos homens entrevistados, no intuito de entender se há diferenças entre estes.

\section{Material e métodos}

A pesquisa faz parte de um estudo maior, que adotou uma abordagem mista: quantitativa, em estudo transversal e descritivo, com banco de dados rodado no programa Stata versão 13.0 e posteriormente analisado pelas pesquisadoras. Foi realizada comparação entre as respostas dos participantes do gênero feminino e masculino com o uso de teste de qui-quadrado. Foi considerado significativo para a análise valores de $\mathrm{p}<0,05$. A parte qualitativa utilizou, para os relatos submetidos ao final do questionário, análise de conteúdo a partir do referencial da pesquisa qualitativa de Bardin.

A porção quantitativa deste estudo foi realizada a partir da aplicação do questionário semiestruturado 'Perfil e sobrecarga dos 
profissionais de saúde e estudantes da área da saúde na Pandemia da Covid-19' em Google Forms, com Termo de Consentimento Livre e Esclarecido, aprovado pelo Comitê de Ética em Pesquisa (CEP) da Santa Casa de Misericórdia de São Paulo, (CAAE: 32682720.8.0000.5479). O questionário foi uma adaptação de outros já validados, utilizados em pesquisas sobre comportamento no Brasil, (saúde mental no Imip Instituto Materno Infantil de Pernambuco e o utilizado pelo coletivo Adelaides: Feminismos e Saúde, no último congresso geral da Abrasco - Associação Brasileira de Saúde Coletiva), por estes tratarem de questões de gênero e saúde. Vale ressaltar que todas as questões respondidas foram analisadas e que nos detivemos mais profundamente naquelas com diferença significativa por gênero, articulando estes achados com a literatura sobre $\mathrm{o}$ assunto. $\mathrm{O}$ questionário foi composto de perguntas de múltipla escolha acerca de dados sociodemográficos, área de atuação, impacto da pandemia nas atividades, contato com o novo coronavírus e diagnóstico de Covid-19, carga de trabalho na pandemia, manifestações e sintomas da sobrecarga (alterações de humor, desconforto físico, alterações cognitivas, agitação, pessimismo, alterações de sono e apetite). Contou com uma porção final livre para os participantes deixarem suas considerações e sugestões por escrito.

Os convites para responder ao questionário foram realizados através da ampla divulgação do endereço eletrônico do questionário em Google Forms, com texto padronizado utilizado pelas pesquisadoras responsáveis, que explicitou os objetivos da pesquisa e convidou as partes interessadas a participarem. A divulgação foi feita a partir da lista de contatos de profissionais da área da saúde das pesquisadoras responsáveis, por meio de ampla divulgação em suas redes. Foram coletadas respostas do dia 22/06/2020 ao dia 07/08/2020, período durante o qual houve constante divulgação do questionário nas redes das pesquisadoras responsáveis. As respostas foram gravadas em base de dados gerada automaticamente e transferidas para um banco de dados virtual.
As perguntas abertas foram transcritas e conferidas de acordo com a fidedignidade.

O banco da pesquisa geral possuiu 386 respostas, das quais 13 eram de participantes que não trabalhavam ou estudavam no estado de São Paulo e foram excluídos da amostra final. Obtivemos então 371 respostas. Para este estudo, foram incluídos apenas os profissionais da área da saúde e, dessa forma, a amostra final foi composta por 149 respostas ao questionário aptas para análise.

Trabalhou-se com amostra de conveniência, composta por profissionais da área da saúde, que se dispuseram a responder o questionário. Por se tratar de amostra de conveniência não probabilística, ressalta-se que os resultados obtidos não podem ser generalizados, entretanto a abordagem dessa população é relevante na compreensão do que as afeta.

Em nosso estudo foram cumpridos os princípios éticos preconizados para pesquisas envolvendo seres humanos.

\section{Resultados}

\section{Caracterização da amostra}

Foram obtidas 149 respostas, dentre elas, 78,5\% do sexo feminino e $21,5 \%$ do sexo masculino. A amostra contou com aproximadamente $40 \%$ dos entrevistados com idade maior que 50 anos, proporção que se mantém entre as mulheres e homens, sendo que, dentre as 117 mulheres, 36 (30,8\%) tinham entre 51 e 60 anos e dentre os 32 homens esse percentual foi de $21,9 \%$, enquanto 11 (9,40\%) mulheres e 21,9\% (7) dos homens tinham mais de 60 anos.

A maior parte dos participantes (53,3\%) apresentou renda média mensal de 8 salários-mínimos ou mais, com uma diferença significativa $(p=0,019)$ entre homens e mulheres. Enquanto entre eles, $75 \%$ recebem 8 salários-mínimos ou mais por mês, entre elas esse número é de apenas $47 \%$. Entre as diferentes categorias profissionais, esse desequilíbrio se 
mantém, exceto entre os enfermeiros, como mostra a tabela 2.

A distribuição das profissões levou em conta a quantidade mínima de pelo menos dez participantes por área de atuação na amostra geral para compor uma categoria. Os demais, foram agrupados na categoria 'outros', essa com alta diversidade de áreas de atuação, representando, dessa forma, o alto percentual de $32,89 \%$. Entretanto, o número de médicos, enfermeiros, psicólogos e nutricionistas foi relevante.

A diferença foi significante $(p=0,002)$ no percentual de homens e mulheres na medicina, sendo a profissão de $56,25 \%$ dos entrevistados sexo masculino e de $19,7 \%$ de entrevistadas do sexo feminino. Do total de médicos da amostra geral, a maioria $(56,1 \%)$ era de mulheres. Deve-se levar em conta que as pesquisadoras do estudo estão inseridas no meio acadêmico e profissional da medicina, sendo atribuída a essa questão possível viés de resultado à amostra, com maior número de participantes da área médica (27,5\%). Entretanto, ressaltam-se outras diferenças como o maior percentual de mulheres que são enfermeiras (17,1\%), psicólogas (18,8\%) e nutricionistas (8,55\%).

Quanto ao tempo de atuação profissional, 69,1\% atuam na profissão há mais de 10 anos, percentual comum entre homens e mulheres. Em relação à área de atuação durante a pandemia, 35,57\% trabalhavam em áreas que não envolviam contato com pacientes, como de gestão e docência. Entretanto, a maior parte da amostra (36,9\%) atuava em contato com pacientes com Covid-19. Entre as mulheres, 32,5\% das entrevistadas estavam em contato com pacientes com Covid-19, entre os homens esse percentual foi de $53,1 \%$ dos 32 entrevistados.

Tabela 1. Caracterização sociodemográfica e profissional da amostra e distribuição de gênero

\begin{tabular}{|c|c|c|c|c|c|c|c|c|}
\hline & \multicolumn{2}{|c|}{ Geral $(n=149)$} & \multicolumn{2}{|c|}{ Feminino $(n=117)$} & \multicolumn{2}{|c|}{ Masculino $(n=32)$} & \multicolumn{2}{|c|}{ Diferença entre sexos } \\
\hline & $\mathbf{n}$ & $\%$ & $\mathbf{n}$ & $\%$ & $\mathbf{n}$ & $\%$ & $\begin{array}{r}\text { Teste de qui- } \\
\text { quadrado }\end{array}$ & $\begin{array}{r}\text { Valor } \\
\text { de } p\end{array}$ \\
\hline \multicolumn{9}{|l|}{ Sexo } \\
\hline Feminino & 117 & $78,52 \%$ & - & - & - & - & & \\
\hline Masculino & 32 & $21,48 \%$ & - & - & - & - & & \\
\hline Idade & & & & & & & 5,9458 & 0,203 \\
\hline $21-30$ & 19 & $12,75 \%$ & 13 & $11,11 \%$ & 6 & $18,75 \%$ & & \\
\hline $31-40$ & 36 & $24,16 \%$ & 29 & $24,79 \%$ & 7 & $21,88 \%$ & & \\
\hline $41-50$ & 33 & $22,15 \%$ & 28 & $23,93 \%$ & 5 & $15,63 \%$ & & \\
\hline $51-60$ & 43 & $28,86 \%$ & 36 & $30,77 \%$ & 7 & $21,88 \%$ & & \\
\hline$>60$ & 18 & $12,08 \%$ & 11 & $9,40 \%$ & 7 & $21,88 \%$ & & \\
\hline Estado civil & & & & & & & 13,1531 & 0,022 \\
\hline Solteiro(a) & 48 & $32,21 \%$ & 36 & $30,77 \%$ & 12 & $37,50 \%$ & & \\
\hline Casado(a) & 60 & $40,27 \%$ & 51 & $43,59 \%$ & 9 & $28,13 \%$ & & \\
\hline Divorciado(a) & 17 & $11,41 \%$ & 16 & $13,68 \%$ & 1 & $3,13 \%$ & & \\
\hline União estável & 22 & $14,77 \%$ & 13 & $11,11 \%$ & 9 & $28,13 \%$ & & \\
\hline Outro & 2 & $1,34 \%$ & 1 & $0,85 \%$ & 1 & $3,13 \%$ & & \\
\hline Renda mensal & & & & & & & 7,9289 & 0,019 \\
\hline Até 4 SM & 24 & $16,11 \%$ & 21 & $17,95 \%$ & 3 & $9,38 \%$ & & \\
\hline 4-8 SM & 46 & $30,87 \%$ & 41 & $35,04 \%$ & 5 & $15,63 \%$ & & \\
\hline $8+S M$ & 79 & $53,02 \%$ & 55 & $47,01 \%$ & 24 & $75,00 \%$ & & \\
\hline
\end{tabular}


Tabela 1. (cont.)

\begin{tabular}{|c|c|c|c|c|c|c|c|c|}
\hline & \multicolumn{2}{|c|}{ Geral $(n=149)$} & \multicolumn{2}{|c|}{ Feminino $(n=117)$} & \multicolumn{2}{|c|}{ Masculino $(n=32)$} & \multicolumn{2}{|c|}{ Diferença entre sexos } \\
\hline & $\mathrm{n}$ & $\%$ & $\mathbf{n}$ & $\%$ & $\mathbf{n}$ & $\%$ & $\begin{array}{r}\text { Teste de qui- } \\
\text { quadrado }\end{array}$ & $\begin{array}{r}\text { Valor } \\
\text { de } p\end{array}$ \\
\hline Profissão & & & & & & & 17,5177 & 0,002 \\
\hline Médico & 41 & $27,52 \%$ & 23 & $19,66 \%$ & 18 & $56,25 \%$ & & \\
\hline Enfermeiro & 24 & $16,11 \%$ & 20 & $17,09 \%$ & 4 & $12,50 \%$ & & \\
\hline Psicólogo & 24 & $16,11 \%$ & 22 & $18,80 \%$ & 2 & $6,25 \%$ & & \\
\hline Nutricionista & 11 & $7,38 \%$ & 10 & $8,55 \%$ & 1 & $3,13 \%$ & & \\
\hline Outros & 49 & $32,89 \%$ & 42 & $35,90 \%$ & 7 & $21,88 \%$ & & \\
\hline Tempo de profissão & & & & & & & 3,6930 & 0,449 \\
\hline Menos de 1 ano & 10 & $6,71 \%$ & 7 & $5,98 \%$ & 3 & $9,38 \%$ & & \\
\hline $1-2$ anos & 7 & $4,70 \%$ & 4 & $3,42 \%$ & 3 & $9,38 \%$ & & \\
\hline 3-5 anos & 10 & $6,71 \%$ & 8 & $6,84 \%$ & 2 & $6,25 \%$ & & \\
\hline 6-10 anos & 19 & $12,75 \%$ & 17 & $14,53 \%$ & 2 & $6,25 \%$ & & \\
\hline Mais de 10 anos & 103 & $69,13 \%$ & 81 & $69,23 \%$ & 22 & $68,75 \%$ & & \\
\hline No momento sua atividade & & & & & & & 6,3762 & 0,095 \\
\hline $\begin{array}{l}\text { Não envolve contato com pa- } \\
\text { cientes }\end{array}$ & 53 & $35,57 \%$ & 42 & $35,90 \%$ & 11 & $34,38 \%$ & & \\
\hline $\begin{array}{l}\text { Envolve contato com pacientes } \\
\text { não Covid-19 }\end{array}$ & 41 & $27,52 \%$ & 37 & $31,62 \%$ & 4 & $12,50 \%$ & & \\
\hline $\begin{array}{l}\text { Envolve contato com pacientes } \\
\text { com Covid-19 }\end{array}$ & 21 & $14,09 \%$ & 14 & $11,97 \%$ & 7 & $21,88 \%$ & & \\
\hline $\begin{array}{l}\text { Envolve contato com pacientes } \\
\text { não Covid-19 e com Covid-19 }\end{array}$ & 34 & $22,82 \%$ & 24 & $20,51 \%$ & 10 & $31,25 \%$ & & \\
\hline
\end{tabular}

Fonte: elaboração própria.

Nota: em negrito valores de $p<0,05$.

Tabela 2. Declaração de renda entre as diferentes categorias profissionais

\begin{tabular}{|c|c|c|c|c|c|c|c|c|c|c|c|c|c|c|c|c|c|c|c|c|}
\hline & \multicolumn{4}{|c|}{ Médico } & \multicolumn{4}{|c|}{ Enfermeiro } & \multicolumn{4}{|c|}{ Psicólogo } & \multicolumn{4}{|c|}{ Nutricionista } & \multicolumn{4}{|c|}{ Outros } \\
\hline & \multicolumn{2}{|c|}{ Homens } & \multicolumn{2}{|c|}{ Mulheres } & \multicolumn{2}{|c|}{ Homens } & \multicolumn{2}{|c|}{ Mulheres } & \multicolumn{2}{|c|}{ Homens } & \multicolumn{2}{|c|}{ Mulheres } & \multicolumn{2}{|c|}{ Homens } & \multicolumn{2}{|c|}{ Mulheres } & \multicolumn{2}{|c|}{ Homens } & \multicolumn{2}{|c|}{ Mulheres } \\
\hline & $\mathrm{n}$ & $\%$ & $\mathrm{n}$ & $\%$ & $n$ & $\%$ & $\mathrm{n}$ & $\%$ & $n$ & $\%$ & $\mathrm{n}$ & $\%$ & $\mathrm{n}$ & $\%$ & $\mathrm{n}$ & $\%$ & n & $\%$ & $\mathrm{n}$ & $\%$ \\
\hline \multicolumn{21}{|c|}{ Renda mensal } \\
\hline Até 4 SM & 1 & $5,56 \%$ & 1 & $4,35 \%$ & 0 & $0,00 \%$ & 3 & $15,00 \%$ & 0 & $0,00 \%$ & 4 & $18,18 \%$ & 0 & $0,00 \%$ & 0 & $0,00 \%$ & 2 & $28,57 \%$ & 13 & $30,95 \%$ \\
\hline $4-8 \mathrm{SM}$ & 0 & $0,00 \%$ & 2 & $8,70 \%$ & 3 & $75,00 \%$ & 10 & $50,00 \%$ & 0 & $0,00 \%$ & 7 & $31,82 \%$ & 0 & $0,00 \%$ & 1 & $10,00 \%$ & 2 & $28,57 \%$ & 21 & $50,00 \%$ \\
\hline $8+S M$ & 17 & $94,44 \%$ & 20 & $86,96 \%$ & 1 & $25,00 \%$ & 7 & $35,00 \%$ & 2 & $100,00 \%$ & 11 & $50,00 \%$ & & $00,00 \%$ & 9 & $90,00 \%$ & 3 & $42,86 \%$ & 8 & $19,05 \%$ \\
\hline
\end{tabular}

Fonte: elaboração própria. 


\section{Impactos da pandemia: avaliação da sobrecarga}

A sobrecarga reportada pelos participantes da pesquisa relativamente às mudanças de vida cotidiana na pandemia foi significativa. 91,3\% do total de entrevistados apontando sobrecarga (gráfico 1). Dentre as $117 \mathrm{mu}-$ lheres entrevistadas, 106 (90,6\%) relataram sentir-se sobrecarregadas e, entre os 32 homens, 30 (93,75\%) relataram o mesmo.

Dos profissionais sobrecarregados, $39,13 \%$ estavam no atendimento de pacientes Covid-19 positivos. Para $47,83 \%$ destes, o trabalho foi o principal fator de cansaço neste contexto (versus. 28,26\% dos profissionais sem contato com pacientes e vs. $23,91 \%$ dos profissionais que atendiam somente pacientes não Covid19). Os profissionais em atendimento de pacientes com Covid-19 foram os que mais reportaram aumento da carga horária dedicada ao trabalho durante a pandemia $(38,54 \%)$, sendo mais da metade $(56,25 \%)$ daqueles que reportam trabalhar $10 \mathrm{~h}$ ou mais horas por dia.

A sobrecarga de trabalho somada ao trabalho doméstico em ambiente domiciliar foi a resposta mais prevalente (48,5\%). Já o percentual de entrevistadas que relataram sobrecarga principalmente em casa, foi de $16,2 \%$. Apenas 9,4\% dos homens entrevistados referiram o mesmo. Quanto aos motivos apontados para a sensação de sobrecarga, ressaltamos que 7 mulheres falam do trabalho doméstico não dividido igualmente como principal fator de cansaço na pandemia e, dentre estas, $4 \mathrm{mu}-$ lheres dizem o mesmo sobre o cuidado com as crianças. Nenhum dos entrevistados do sexo masculino, apontou o mesmo. O consumo constante de informações sobre a pandemia foi o fator de cansaço mais lembrado por ambos: homens e mulheres.

Gráfico 1. Sobrecarga em decorrência da pandemia e fator apontado como principal motivo desse cansaço em meio à sobrecarga relatada

Apresentação de sobrecarga em decorrência da pandemia

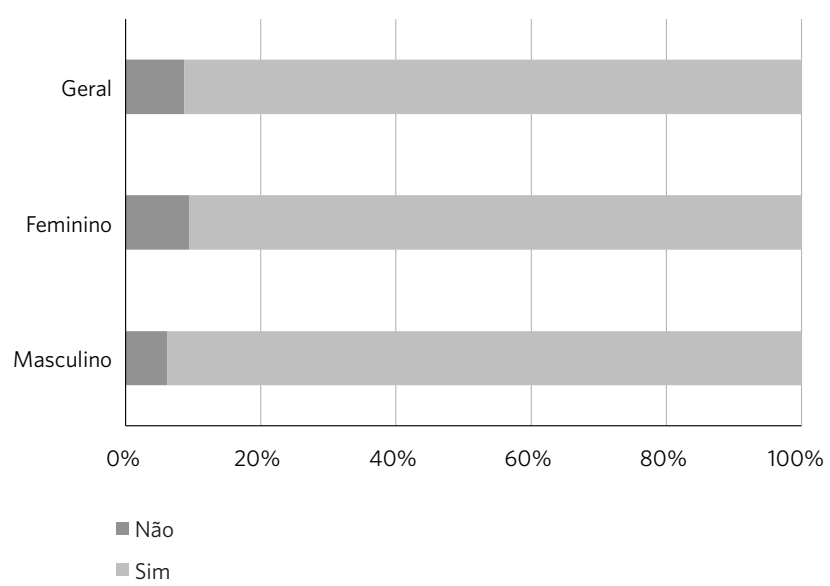

Fator de maior cansaço em meio à pandemia

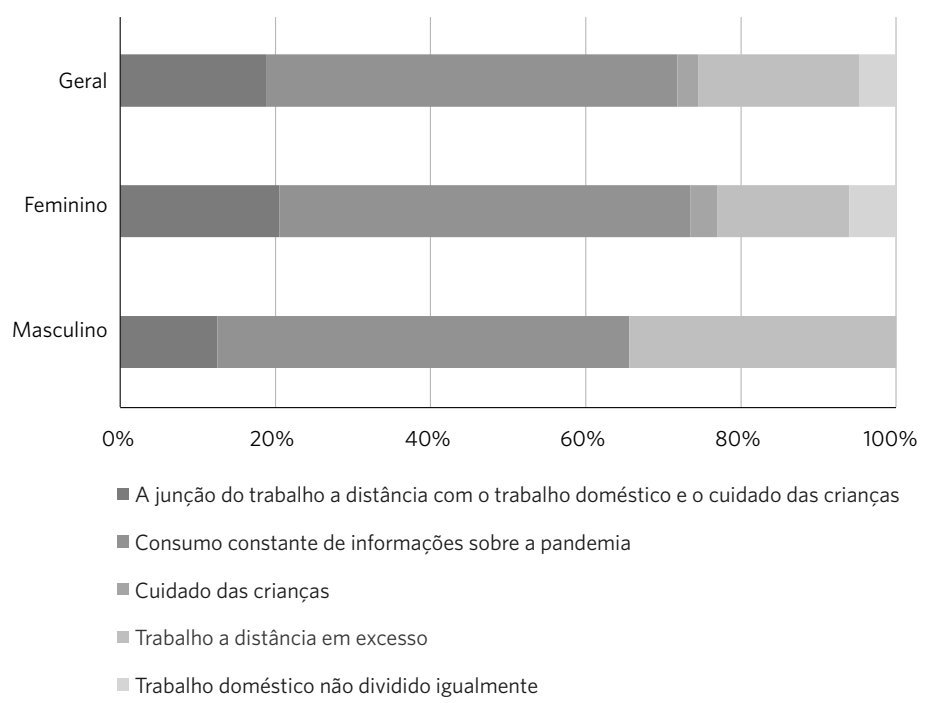


Quanto às manifestações ou sintomas da sobrecarga, $70,5 \%$ da amostra geral referiu alterações no humor como mau humor, irritabilidade e desânimo, esse percentual foi o mesmo entre homens e mulheres. Mais da metade $(57,3 \%)$ das mulheres e metade $(50 \%)$ dos homens referiu desconforto físico (dor de cabeça, dor muscular, cansaço, enjoos, tontura). Enquanto 41,9\% das mulheres referiram problemas de memória, dificuldade em concentrar-se, confusão, e 39,3\% agitação e inquietação, respectivamente, somente $21,9 \%$ e $12 \%$ dos homens reportaram o mesmo. O pessimismo excessivo foi mais reportado pelos homens $(31,25 \%)$ do que pelas mulheres $(23,1 \%)$. As alterações de sono foram notadas por mais da metade da amostra total $(53,69 \%)$, sendo que um quarto das mulheres $(25,6 \%)$ e um oitavo dos homens $(12,5 \%)$ relataram aumento na quantidade de pesadelos.

\section{Ambiente familiar}

Houve uma diferença significativa $(p=0,022)$ de estado civil entre homens e mulheres. Quando analisada a amostra geral, a maior parte (40,3\%) era de pessoas casadas. Entretanto, esse percentual foi maior entre as mulheres $(43,6 \%)$ do que entre os homens (28,1\%).

A maior parte dos homens (37,5\%) e quase um terço das mulheres (30,8\%) era solteiro(a). Somente $11,4 \%$ da amostra era divorciada. A maior parte dos divorciados estavam entre as mulheres (13,7\%). Homens eram 3,1\%. Um percentual considerável de participantes tinha união estável (14,8\%), mais frequente entre os homens $(28,1 \%)$.

Gráfico 2. Aumento do trabalho doméstico e de cuidados no contexto da pandemia na amostra geral, entre os homens da amostra e entre as mulheres da amostra

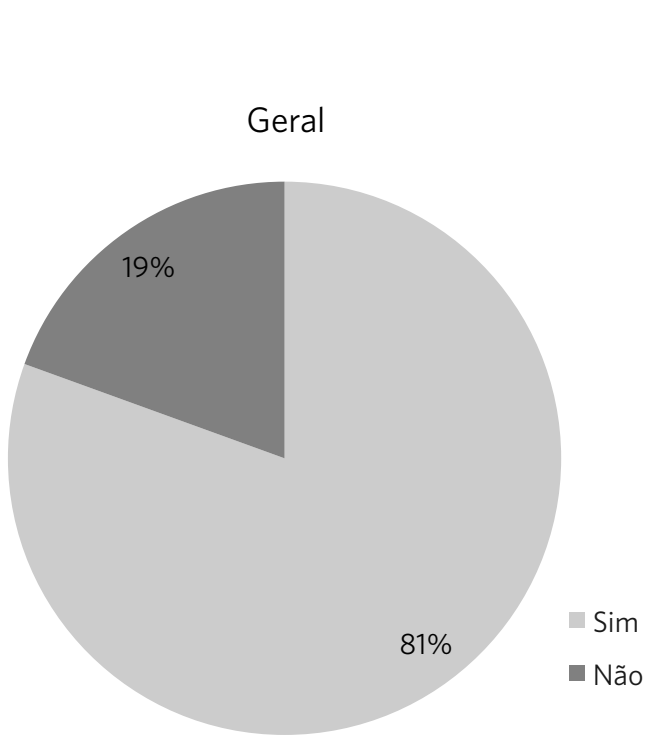

Mulheres

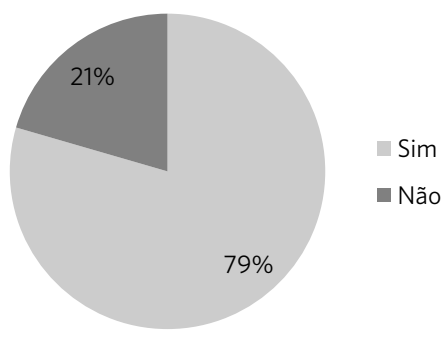

Homens

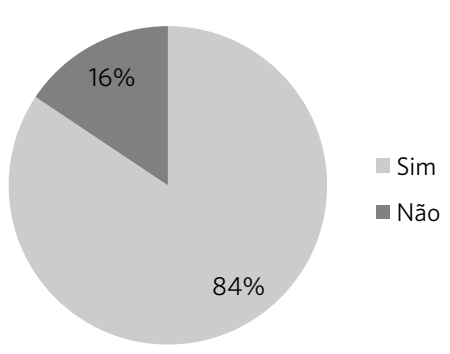

Fonte: elaboração própria. 
Em relação aos trabalhos domésticos, a maior parte dos entrevistados (80,5\%) apontou que estes aumentaram muito no contexto da pandemia (gráfico 2). Esse percentual foi de 79,5\% entre as 117 mulheres entrevistadas e 84,4\% entre os 32 homens. Já a responsabilidade pelo trabalho doméstico foi apontada majoritariamente como do entrevistado. Entretanto, enquanto mais de dois terços $(69,2 \%)$ das mulheres referem ser responsáveis pelo trabalho doméstico em sua residência, nem metade (46,9\%) dos homens refere o mesmo. Também, um percentual maior de homens, $15,6 \%$ versus $6 \%$, refere depender de mulheres como a mãe ou empregada doméstica/diarista na realização dos trabalhos domésticos.

Gráfico 3. Responsável pelo trabalho doméstico na residência do(a) entrevistado(a)

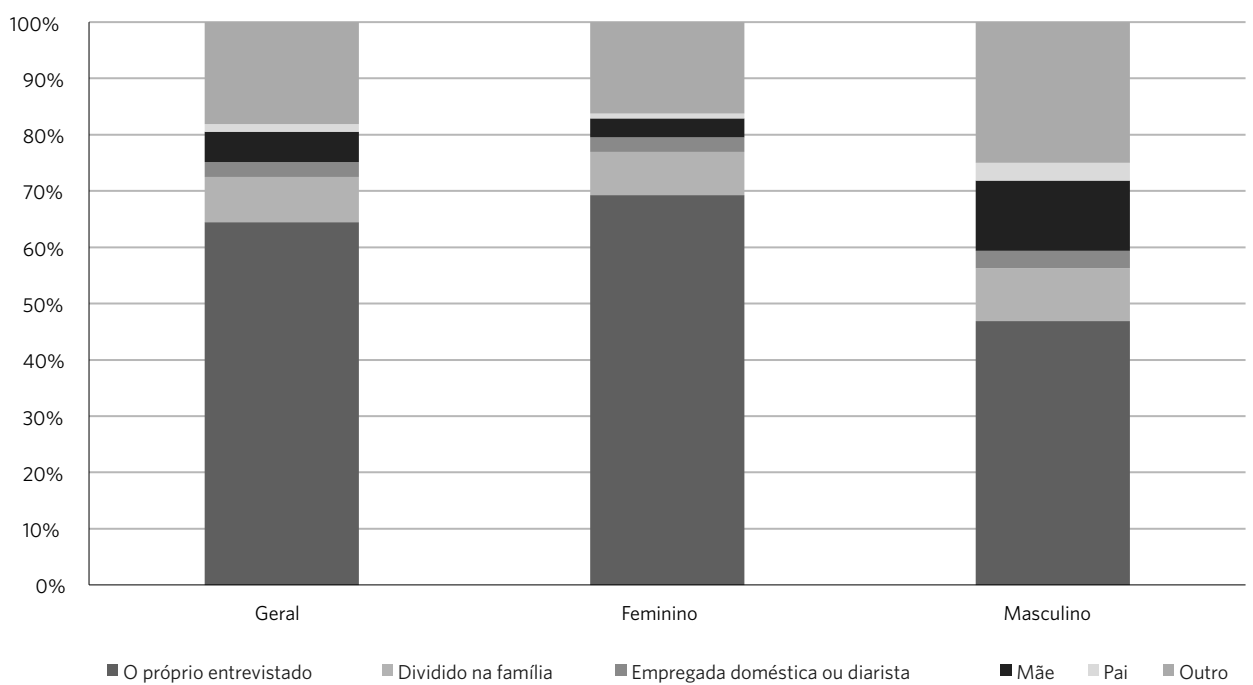

Fonte: elaboração própria.

\section{Discussão}

Os resultados que encontramos corroboram com a literatura nacional e internacional sobre a participação das mulheres na área da saúde: elas são maioria ${ }^{15,16}$. A presença maior de homens que de mulheres na medicina também é apontada pela literatura. Pesquisadores da Faculdade de Medicina da Universidade de São Paulo (USP), em 2017 , apontaram a participação de $54,4 \%$ de homens, comparados aos $45,6 \%$ de mulheres na profissão ${ }^{16}$. Entretanto, temos referências na literatura de que está em curso um processo de 'feminização' da medicina, que também é demonstrada em nossa amostra com maioria feminina entre os médicos do estudo. A tendência de maior participação feminina na carreira médica também aparece entre as mais jovens, com o maior número de mulheres entre os estudantes da graduação ${ }^{16}$.

Entre as outras categorias profissionais, a diferença entre participação de homens e mulheres não se mantém. Historicamente, trabalhos de cuidados relacionados a áreas como a da enfermagem, parte importante de nossa amostra, já eram ocupados majoritariamente por mulheres, enquanto os homens ocupavam áreas de maior prestígio social, como a medicina ${ }^{15}$. 
Achado importante foi a diferença entre a renda média mensal referida pelas mulheres em relação à dos homens. Parte da literatura aponta que, no geral e na área da saúde, mulheres ganham menos que os homens mesmo que possuam a mesma formação ${ }^{\mathbf{1 6}, 28,29}$. Dados da OMS apontam que, mesmo que a hora trabalho seja a mesma, a desigualdade salarial entre homens e mulheres na mesma profissão está presente e aparece na composição da renda mensal. São eles que, na maior parte das vezes, acumulam os cargos de chefia, coordenação e diretoria, o que amplia o salário final. Os homens possuem também maior atuação na área do ensino, na cirurgia e na administração $0^{30}$ - áreas de maior prestígio social - estando mais frequentemente em posições de poder e de maior renda mensal.

Segundo a OMS, mesmo após descontada a diferença de horas trabalhadas e as diferentes ocupações entre homens e mulheres, ainda há um abismo inexplicável de $11,2 \%$ entre o salário dos homens e mulheres dentro da mesma ocupação e trabalhando a mesma quantidade de horas ${ }^{15}$.

As mulheres, por sua vez, têm maior papel no trabalho não remunerado realizado em casa (trabalho doméstico, cuidado com as crianças etc.) que, além de diminuir sua disponibilidade de tempo para acumulação de cargos semelhante aos homens, as desgasta sobremaneira. Ainda, sustentado por Fraser, esse trabalho reprodutivo realizado por elas é parte do que sustenta o trabalho produtivo realizado pelos homens e que os permite maior acúmulo de renda, perpetuando a desigualda$\mathrm{de}^{22,30}$. A literatura aponta que são as mulheres que, em meio a pandemia, mais reduziram o horário no trabalho e, consequentemente, sua renda, principalmente aquelas com filhos e em casais que ambos trabalham ${ }^{31}$. A desigualdade entre homens e mulheres frente ao trabalho e renda é uma dentre as várias situações exacerbadas pela pandemia. Não obstante, para muitas profissionais da saúde, essa redução não foi possível e, ainda assim, o acúmulo de atividades domésticas foi enorme.
A maior parte de nossa amostra (36,9\%) trabalha diretamente com pacientes com síndrome gripal/sintomáticos respiratórios/ positivos para a Covid-19 em suas rotinas de trabalho. Nossos dados apontam que estes profissionais são os que estiveram submetidos à maior carga de trabalho no contexto da pandemia. Alguns estudos apontam ainda que os profissionais de saúde em contato direto com pacientes positivos para Covid-19 apresentam mais frequentemente sintomas de ansiedade, depressão, perda da qualidade do sono e angústia $^{32}$. Grande parte da nossa amostra $(35,9 \%)$ relatou não trabalhar em contato direto com a área Covid-19, mas em atividades de gestão, pesquisa ou docência. Isso pode ser um viés de resultado em decorrência da área de atuação de uma das pesquisadoras responsáveis como docente e pesquisadora. Entretanto, a saúde mental de todos os profissionais da área da saúde foi impactada como consequência dos desafios proporcionados pela pandemia e exige olhar singular ${ }^{33,34}$.

É de extrema relevância a sobrecarga descrita pelos participantes da pesquisa. Passados dezenove meses de pandemia, os dados sobre a Covid-19 pelo consórcio de veículos de imprensa indicam média móvel de casos de Covid-19 acima de 60 mil e de mortes pela doença acima de 1500 desde março até julho de 2021. Neste tempo, acumularam-se cansaço, ansiedade, estresse e trabalho na vida dos profissionais da saúde, que permanecem os mesmos desde o início. O impacto na saúde mental desses profissionais é real ${ }^{25,32}$ e continuará afetando sua atuação e sua saúde, caso não haja um olhar específico para tais trabalhadores.

As manifestações físicas e emocionais da sobrecarga estiveram bastante presentes, tanto entre os homens quanto entre as mulheres, reforçando o impacto negativo já evidenciado da pandemia na saúde mental dos profissionais da saúde ${ }^{32,34}$. Nota-se que as mulheres reportaram mais desconforto físico, alterações cognitivas, agitação e inquietação e maior aumento de pesadelos, ou seja, sua saúde mental se encontra mais fragilizada. 
$\mathrm{Na}$ literatura, alguns dos principais fatores estressores numa quarentena advinda de uma pandemia são: duração da quarentena, medo de infecção, frustração e tédio, suprimentos básicos inadequados e informação inadequada sobre a pandemia ${ }^{36}$. Se considerarmos o contexto brasileiro, mesmo que aqui o conceito de quarentena diferencie isolamento e distanciamento social, alguns aspectos avaliados são aplicáveis à realidade do País como fatores estressores no período da pandemia da Covid-19: a incerteza da duração do isolamento social, o medo de infecção, a frustração e tédio ao permanecer longos períodos afastados da sociedade e, por fim, a falta e contradição de informações fornecidas pelos líderes de governo que fazem parte da rotina dos brasileiros e, assim, dos profissionais da saúde ${ }^{5}$. A literatura apoia que esses fatores repercutem diferentemente conforme o gênero ${ }^{37}$.

Ao analisar os fatores de cansaço geradores de sobrecarga entre mulheres e homens, algumas noções permaneceram semelhantes, independente do gênero. Há alta relevância do consumo constante de informações sobre a pandemia (a razão de cansaço mais prevalente para ambos) e o trabalho a distância em excesso como geradores de sobrecarga. De acordo com a literatura, o consumo de noticiários e notícias mais densos, rotineiro no cenário atual, está associado com pior saúde mental e bem-estar ${ }^{38}$. Vale ressaltar que a parcela de mulheres que se sentem cansadas em decorrência da junção do trabalho à distância com o trabalho doméstico e o cuidado das crianças foi maior que a parcela de homens. Isso corrobora a literatura que aponta que as mulheres estão submetidas à dupla jornada de trabalho, absorvendo o peso do trabalho doméstico mesmo no contexto atual ${ }^{39}$.

Enquanto parte das mulheres reportaram o trabalho doméstico não dividido igualmente ou o cuidado com as crianças como o fator principal de cansaço, nenhum homem sequer selecionou esta alternativa. Considerando o papel social da mulher como principal determinante na atribuição do trabalho doméstico não remunerado e a consequente divisão desequilibrada desse nas famílias brasileiras ${ }^{40}$, os achados da pesquisa reforçam as desigualdades nos papéis de gênero postas em discussão por Beauvoir, Hirata e Péres ${ }^{17-19}$. O cuidado com o lar, com as crianças e animais é invisibilizado na vida de mulheres trabalhadoras que, mesmo após um longo dia de trabalho, chegam em casa e se deparam com funções e afazeres não atribuídos aos homens do lar.

A maior parte da amostra considera que houve aumento do trabalho doméstico durante a pandemia. Este aumento foi mais percebido pelos homens do que pelas mulheres. Isso pode ser em decorrência de um aumento em relação ao que costumavam fazer, pois previamente à pandemia, o trabalho doméstico já era majoritariamente realizado por mulheres do $\operatorname{lar}^{\mathbf{2 0}}$ ou era terceirizado para uma empregada doméstica ${ }^{23}$. Com a alteração das relações interpessoais e dinâmicas organizacionais decorrentes da pandemia, é possível que tenha havido melhor divisão do trabalho doméstico em algumas residências, sendo notado mais pelos homens. Outra possibilidade, se baseia na concepção de que a realização do trabalho doméstico pelas mulheres já está naturalizada dentro do sistema patriarcal ${ }^{18}$, visto que dados coletados em março de 2021 pelo Núcleo de Estudos da Burocracia (Fundação Getulio Vargas - FGV) apontam que elas acumularam mais de 30 horas semanais de atividades domésticas durante a pandemia ${ }^{39}$.

Consequentemente, as mulheres nem mesmo reconhecem este aumento ou até mesmo a sobrecarga que ele gera. Nossos dados apontam que mais de $60 \%$ das mulheres entrevistadas eram as responsáveis pelo trabalho doméstico em sua residência enquanto nem metade dos homens possuíam a mesma incumbência. Podemos notar também o maior volume de trabalho doméstico nas residências masculinas que são terceirizadas para outras mulheres, como mães e empregadas domésticas, o que reforça a divisão sexual do trabalho enraizada na sociedade brasileira ${ }^{\mathbf{1 8}}$. A sobrecarga de trabalho para as mulheres 
acaba sendo, portanto, no âmbito profissional e doméstico. Consequência disso parece ser a maior carga mental e aumento da suscetibilidade ao adoecimento mental. A literatura mostra que as mulheres da área da saúde reportam mais exaustão mental e manifestam mais sintomas da síndrome de Burnout que os homens ${ }^{41}$.

\section{Limitações}

Quanto às limitações do estudo, há limitação do espaço amostral e da generalização possível a partir dos dados e resultados obtidos pelo tipo de amostra utilizada. Além disso, apesar do número relevante de respostas analisadas, houve grande heterogeneidade de atuação profissional reportada, tanto referente à profissão como em relação ao atendimento ou não de pacientes. Esses grupos possuem realidades diferentes e difíceis de comparar.

Apesar disso, o estudo apresenta vários pontos fortes. Traz um olhar sobre a situação de um grupo muito relevante no enfrentamento à pandemia: os profissionais da saúde. Independente do grau de contato com a Covid19 , todos atuam direta ou indiretamente na pandemia. Ademais, traz um olhar de gênero, ainda pouco empregado sobre esta população, apontando dificuldades enfrentadas pelas mulheres, muitas vezes negligenciadas na nossa sociedade patriarcal.

\section{Conclusões}

A realidade brasileira foi determinante ao avaliar o impacto da pandemia na vida dos trabalhadores da saúde, segundo nosso estudo. Os profissionais da saúde já são um grupo vulnerável no contexto atual e compreendemos que, ainda por cima, há fatores descritos como estressores presentes cotidianamente na rotina dos entrevistados, como a incerteza do tempo de isolamento, o medo da infecção, a frustração ao enfrentar desfechos desfavoráveis de seus pacientes, assim como a falta de apoio das políticas públicas adequadas ao enfrentamento da pandemia e a contradição das informações fornecidas pelos líderes de governo.

Além de evidenciar a sobrecarga relatada pelos profissionais da saúde, um dos pilares do combate ao novo coronavírus, nosso estudo demonstrou evidente diferenças de gênero nos impactos da pandemia. Se enquanto profissionais as mulheres sofreram o mesmo impacto que os homens, ao analisarmos suas realidades neste contexto, a diferença nos fatores que geraram sobrecarga para as mulheres, ligados ao ambiente doméstico, a baixa remuneração para desenvolver as mesmas funções e seu papel de gênero, reflete em maior fragilização de sua saúde mental, evidenciada pelos resultados obtidos. Nosso estudo concluiu, portanto, que a pandemia da Covid-19 é mais difícil para elas.

Em cenário sem precedentes, ainda não é possível saber as repercussões futuras desse fato, nem se esta situação será considerada futuramente uma das sequelas da pandemia. É importante utilizarmos o olhar de gênero ao pensar estratégias de intervenção para recuperação saudável da sociedade em um futuro pós-pandêmico no qual as consequências de uma sociedade sobrecarregada virão.

\section{Colaboradoras}

Vieira J (0000-0003-2360-1186)* contribuiu para a realização da pesquisa com aplicação do questionário, coleta e análise de dados, revisão bibliográfica, elaboração e revisão do manuscrito. Anido I (0000-0002-3058-2107)* contribuiu para a realização da pesquisa com aplicação do questionário, coleta de dados e revisão do manuscrito. Calife K (0000-00027038-8069)* contribuiu para a realização da pesquisa, elaboração e revisão do manuscrito. 


\section{Referências}

1. World Health Organization. WHO Director General's Opening Remarks at the Media Briefing on COVID 19 - 11 March 2020. 2020. [acesso em 2020 set 20]. Disponível em: https://www.who.int/director-general/ speeches/detail/who-director-general-s-opening-remarks-at-the-media-briefing-on-covid-19---11-march-2020.

2. World Health Organization. Coronavirus disease (COVID-19) advice for the public. 2021. [acesso em 2021 jun 25]. Disponível em: https://www.who.int/ emergencies/diseases/novel-coronavirus-2019/advice-for-public.

3. Cousins S. New Zealand eliminates COVID-19. Lancet. 2020; 395(10235):1474.

4. Dong E, Du H, Gardner L. An interactive web-based dashboard to track COVID-19 in real time. Lancet infect. diseases. 2020; 20(5):533-4.

5. Lancet T. COVID-19 in Brazil: "So what?". Lancet. 2020; 395(10235):1461.

6. Pfefferbaum B, North CS. Mental health and the Covid-19 pandemic. N Engl J Med. 2020; (383):510-512.

7. Calife K, Chioro A. A pandemia da Covid-19 em São Paulo: o caminho da desigualdade. 2020. [acesso em 2020 nov 24]. Disponível em: https://fpabramo.org. br/observabr/2020/11/19/a-pandemia-da-covid-19-em-sao-paulo-o-caminho-da-desigualdade/.

8. Chioro A, Calife K, Santos Barros CR, et al. COVID-19 em uma Região Metropolitana: vulnerabilidade social e políticas públicas em contextos de desigualdades. Saúde debate. 2020; 44(esp4):219-231.

9. Rex FE, Borges CAS, Kafer PS. Spatial analysis of the COVID-19 distribution pattern in São Paulo State, Brazil. Ciênc. Saúde Colet. 2020 [acesso em 2020 nov 24]; 25(9). Disponível em: http://www.cienciaesaudecoletiva.com.br/artigos/spatial-analysis-of-the-covid19-distribution-pattern-in-sao-paulo-state-brazil $/ 17603$ ?id= 17603 .
10. Tess B, Alves MC, Reinach F, et al. Inquérito domiciliar para monitorar a soroprevalência da infecção pelo vírus SARS-CoV-2 em adultos no município de São Paulo entre os dias 1 e 10 de outubro de 2020. SoroEpi MSP. 2020. [acesso em 2020 nov 24]. Disponível em: https://www.monitoramentocovid19.org.

11. World Health Organization. Coronavirus disease (COVID-19): Weekly epidemiological, update - 17 November 2020. 2020. [acesso em $2020 \mathrm{dez} 15$ ]. Disponível em: https://www.who.int/docs/default-source/coronaviruse/situation-reports/weekly-epi-update-14.pd f?sfvrsn=df2b8dd_6\&download=true.

12. Fundação Oswaldo Cruz. Boletim Extraordinário 14 de julho de 2021. Boletim Observatório Covid-19. 2021. [acesso em 2021 ago 5]. Disponível em: https:// portal.fiocruz.br/sites/portal.fiocruz.br/files/documentos/boletim_extraordinario_2021-julho.pdf.

13. Mathieu E, Ritchie H, Ortiz-Ospina E, et al. A global database of COVID-19 vaccinations. Nat Hum Behav. 2021.

14. Lima NT, Buss PM, Paes-Sousa R. COVID-19 pandemic: A health and humanitarian crisis. Cad. Saúde Pública. 2020; 36(7).

15. Boniol M, McIsaac M, Xu L, et al. Gender equity in the health workforce: analysis of 104 countries. Geneva: World Health Organization; 2019. [acesso em 2020 ago 15]. Disponível em: https://apps.who.int/iris/bitstream/handle/10665/311314/WHO-HIS-HWF-Gender-WP1-2019.1-eng.pdf?sequence=1\&isAllowed=y.

16. Scheffer M, Cassenote A, Guilloux AGA, et al. Demografia Médica no Brasil. 2018. São Paulo: FMUSP; CFM; Cremesp; 2018.

17. De Beauvoir S. O segundo sexo. Rio de Janeiro: Nova Fronteira; 2014.

18. Hirata H, Kergoat D. Novas configurações da divisão sexual do trabalho. Cad. de pesq. 2007; 37(132):595609. 
19. Peres L. Os desafios para o século XXI. Mulher e Trabalho. 2011; (1):51-53.

20. Instituto Brasileiro de Geografia e Estatística. Em média, mulheres dedicam 10,4 horas por semana a mais que os homens aos afazeres domésticos ou ao cuidado de pessoas. 2020. [acesso em 2021 jun 25]. Disponível em: https://agenciadenoticias.ibge.gov.br/ agencia-sala-de-imprensa/2013-agencia-de-noticias/ releases/27877-em-media-mulheres-dedicam-10-4-horas-por-semana-a-mais-que-os-homens-aos-afazeres-domesticos-ou-ao-cuidado-de-pessoas.

21. Vogel L. Marxism and the oppression of women: Toward a unitary theory. Chicago: Haymarket Books; 1983.

22. Fraser N. Feminismo, capitalismo e a astúcia da história. In: Hollanda HB, organizadora. Pensamento feminista. Conceitos fundamentais. Rio de Janeiro: Bazar do Tempo; 2019.

23. Brites J. Afeto e desigualdade: gênero, geração e classe entre empregadas domésticas e seus empregadores. Cadernos pagu. 2007; (29):91-109.

24. Gausman J, Langer A. Sex and gender disparities in the COVID-19 pandemic. J. of Women's Health. 2020; 29(4):465-6.

25. Spoorthy MS, Pratapa SK, Mahant S. Mental health problems faced by healthcare workers due to the COVID-19 pandemic-A review. Asian J. psyc. 2020; (51):102119.

26. Ornell F, Schuch JB, Sordi AO, et al. "Pandemic fear" and COVID-19: mental health burden and strategies. Braz. J. Psyc. 2020; 42(3):232-5.

27. Organização Mundial da Saúde, Organização Pan-Americana da Saúde. (OPAS/OMS). Serviços Recomendações Gerais de Saúde. Proteção da saúde mental em situações de epidemias. 2006. [acesso em 2021 jun 20]. Disponível: https://www.paho.org/hq/dmdocuments/2009/Protecao-da-Saude-Mental-em-Situaciones-de-Epidemias--Portugues.pdf.
28. Probst ER, Ramos P. A evolução da mulher no mercado de trabalho. Santa Catarina: ICPG; 2003.

29. Mahase E. Gender pay gap in general practice is $35 \%$, report finds-owing to age, working hours, and partnerships. BMJ. 2020; (368):m112.

30. Santos TS. Gênero e carreira profissional na Medicina. Mulher e trabalho. 2011; (21):4.

31. Collins C, Landivar LC, Ruppanner L, et al. COVID 19 and the gender gap in work hours. Gender Work Organ. 2021; 28(S1):101-112.

32. Lai J, Ma S, Wang Y, et al. Factors Associated With Mental Health Outcomes Among Health Care Workers Exposed to Coronavirus Disease. JAMA Netw Open. 2020; 3(3):e203976.

33. Fundação Oswaldo Cruz. Cartilha de Recomendações Gerais: Saúde Mental e Atenção Psicossocial na Pandemia de Covid-19. 2020. [acesso em 2021 jun 25]. Disponível em: https://portal.fiocruz.br/sites/portal. fiocruz.br/files/documentos/cartilha_recomendacoes_gerais_06_04_0.pdf.

34. Teixeira CFS, Soares CM, Souza EA, et al. A saúde dos profissionais de saúde no enfrentamento da pandemia de Covid-19. Ciênc. Saúde Colet. 2020 [acesso em 2021 jul 9]; 25(9):3465-3474. Disponível em: https://doi.org/10.1590/1413-81232020259.19562020.

35. Lotta G, Fernandez M, Magri G, et al. A pandemia de COVID19 e (os)as profissionais de saúde pública: uma perspectiva de gênero e raça sobre a linha de frente. Núcleo de Estudos da Burocracia e Fiocruz. 2021. [acesso em 2021 jul 9]. Disponível em: https:// neburocracia.wordpress.com/publicacoes/.

36. Brooks SK, Webster RK, Smith LE, et al. The psychological impact of quarantine and how to reduce it: rapid review of the evidence. The Lancet. 2020; 395(10227):913-920.

37. Lotta G, Fernandez M, Magri G, et al. A pandemia de COVID19 e (os)as profissionais de saúde pública: 
uma perspectiva de gênero e raça sobre a linha de frente. Núcleo de Estudos da Burocracia e Fiocruz. 2021. [acesso em 2021 jul 9]. Disponível em: https:// neburocracia.wordpress.com/publicacoes/.

38. Boukes M, Vliegenthart R. News consumption and its unpleasant side effect. J. Med. Psyc. 2017; 29(3):137147.

39. Lotta G, Fernandez M, Corrêa M, et al. A pandemia de COVID19 e (os)as profissionais de saúde pública: $2^{\text {a }}$ fase. Núcleo de Estudos da Burocracia e Fiocruz. 2021. [acesso em 2021 jul 9]. Disponível em: https:// neburocracia.wordpress.com/publicacoes/.
40. Melo HP, Castilho M. Trabalho reprodutivo no Brasil: quem faz?. Rev econ. contemp. 2009; 13(1):135-58.

41. Barello S, Palamenghi L, Graffigna G. Burnout and somatic symptoms among frontline healthcare professionals at the peak of the Italian COVID-19 pandemic. Psych. Res. 2020; (290):113129.

Recebido em 16/07/2021

Aprovado em 04/11/2021

Conflito de interesses: inexistente

Suporte financeiro: não houve 